\title{
ADAPTAÇÃO DE FORNO DE MICROONDAS DOMÉSTICO PARA REALIZAÇÃo DE REAÇÕES DE TRANSESTERIFICAÇÃO SOB REFLUXO E CATÁLISE POR ARGILAS
}

\author{
Fernando de C. da Silva, Vitor F. Ferreira* e Maria Cecília B. V. de Souza \\ Departamento de Química Orgânica, Instituto de Química, Universidade Federal Fluminense, Outeiro de São João Batista, s/n, \\ 24020-150 Niterói - RJ
}

Recebido em 7/3/05; aceito em 22/7/05; publicado na web em 20/1/06

\begin{abstract}
ADAPTING A DOMESTIC MICROWAVE OVEN TRANSESTERIFICATION REACTIONS FOR UNDER REFLUX AND CLAY CATALYSIS. The microwave oven became an important source of heating for many laboratory procedures including accelerating organic reactions. Reactions that require long reflux times can sometimes be carried out in a few hours or minutes in a conventional microwave oven. However, longer reflux times can be troublesome since domestic microwave ovens are not prepared for these harsh conditions. This technical note presents our finding on heterogeneous catalysis transesterification reactions between b-ketoesters and carbohydrate derivatives under heating or microwave irradiation using an adapted domestic microwave oven.
\end{abstract}

Keywords: microwave; transesterification; clay catalysis

\section{INTRODUÇÃO}

Nos dias atuais nos quais as demandas por processos sintéticos mais limpos e eficientes têm sido consideradas muito relevantes devido a aspectos ambientais, duas condições reacionais parecem bastante adequadas para as reações químicas em escala preparativa: reações livres de solventes e reações aceleradas sob irradiação de microondas (MO). Neste sentido, devido à eficiência de aparelhos de $\mathrm{MO}$ em aquecer rapidamente meios reacionais, estes têm sido muito utilizados em diversas transformações orgânicas reduzindo seus tempos de realizações, muitas vezes de dias e horas para minutos ou segundos. Revisões recentes de artigos publicados em periódicos de nível científico reconhecido sobre reações aceleradas por $\mathrm{MO}^{2-5}$ mostram que é grande a diversidade de tipos de reações beneficiadas por esta espécie de aquecimento ${ }^{6}$.

Os fornos de MO também podem ser utilizados para muitos outros fins nos laboratórios de química ${ }^{7}$. Evidentemente, já existem no mercado internacional diferentes fornos de MO que se adaptam a diversas finalidades, porém ainda com preços elevados que impossibilitam seu uso como equipamento de rotina em laboratório. Assim, muitos pesquisadores têm optado por desenvolver projetos próprios de fabricação de fornos de $\mathrm{MO}^{8}$ ou utilizar fornos domésticos para fins laboratoriais, com ou sem adaptações 9 .

Manter reações sob refluxo por longo período de tempo ( $>24$ h) por métodos convencionais é uma operação laboratorial freqüentemente utilizada. $\mathrm{O}$ uso de fornos de $\mathrm{MO}$, adaptados ou não, para reações com estas características, encontra-se ainda limitado por motivos técnicos, principalmente no que diz respeito ao tempo de vida útil do magnetron, componente mais caro do forno, responsável por gerar as MO.

Reações que utilizam catalisadores inorgânicos sólidos que possam ser regenerados representam um avanço no campo da química ambientalmente responsável. As argilas são materiais naturais, terrosos e de granulação fina que podem ser utilizados como catalisadores sólidos para muitas reações orgânicas. Devido à necessidade de se enfatizar o desenvolvimento de processos químicos

*e-mail: cegvito@vm.uff.br menos danosos ao ambiente e na busca de otimização de processos que envolvam a substituição em reações de ácidos e bases aquosas por catalisadores sólidos, há um grande interesse no estudo ou no desenvolvimento de novos catalisadores que tenham como base sólidos inorgânicos. O uso de argilas como catalisadores tem merecido atenção nas áreas de química orgânica, incluindo-se a síntese orgânica, principalmente no que se relaciona à quimio e regiosseletividade, ao baixo custo de materiais, às condições reacionais brandas, à sua fácil obtenção e possibilidade de sua reutilização ${ }^{10-13}$.

As reações de transesterificação têm recebido maior destaque mais recentemente devido à demanda por biodiesel, incentivada pelo programa nacional de combustíveis alternativos derivados de óleos vegetais. Estas reações também são importantes do ponto de vista preparativo ${ }^{14}$, pois ésteres complexos podem ser obtidos a partir de outros mais simples. Este processo normalmente é realizado sob temperaturas elevadas e por longos tempos reacionais (>24 h). O uso de catalisadores heterogêneos, como argilas e outros sólidos inorgânicos, leva à diminuição deste tempo de reação ${ }^{15}$. Assim, a transferência de calor por MO e o emprego de argilas são fatores que podem resultar em condições bastante adequadas para redução dos tempos ${ }^{16}$.

Este trabalho tem por objetivo adaptar um forno de microondas doméstico de modo que possa vir a ser utilizado por longos períodos de tempo em reações de transesterificação ${ }^{17}$. O efeito da irradiação de MO sobre os tempos das reações de transesterificação será avaliado usando-se como modelo a reação entre $\beta$-cetoésteres $(\mathbf{1 , 2})$ e acetonídeos (3a-f) sob catálise de argilas naturais de três tipos: esmectita, atapulgita e vermiculita.

\section{RESULTADOS E DISCUSSÃO}

\section{Adaptação de forno de microondas doméstico para realização de reações sob refluxo e catálise por argilas}

Os primeiros testes realizados com o equipamento adaptado indicaram que as reações de transesterificação ocorreriam com um tempo entre 7-9 h, muito menor que o necessário para realizar estas mesmas nas reações sob aquecimento convencional (48 h).

Mesmo com as adaptações para resfriamento do magnetron, 
pode haver danos irreversíveis ao mesmo, devido à baixa absorção das microondas excedentes e das não absorvidas na reação, principalmente por solvente de baixa polaridade (ex. tolueno). Sabendose que a freqüência do aparelho é de $2450 \mathrm{MHz}$ e que solventes polares, em especial a água, absorvem bem este tipo de radiação, foi confeccionado um tubo de vidro em forma de "U" (Figura 1), pelo qual se fez passar água resfriada externamente para captação das MO excedentes. Essa absorção pode ser regulada na medida em que se aumente ou diminua o volume de água no tubo ou que o mesmo avance ou recue em relação à face posterior do forno. Este tubo mostrou-se de suma importância para a vida útil do magnetron, uma vez que nas reações utilizou-se tolueno, solvente de baixa polaridade. A introdução do tubo em "U" para moderação das MO no interior do forno já havia sido descrita por Hogarth e colaboradores $^{18}$ para reações sob refluxo. Entretanto, no nosso caso o tubo se localiza na face posterior do forno, como já mencionado, próximo ao fundo do mesmo, permitindo a circulação de água no ponto de maior focalização das ondas.

\section{(a)}

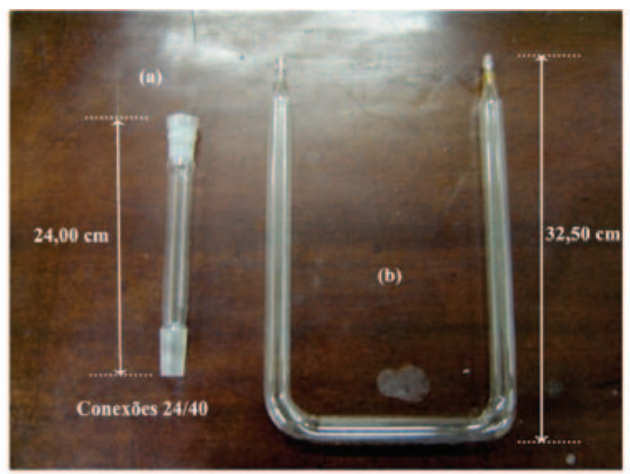

(b)

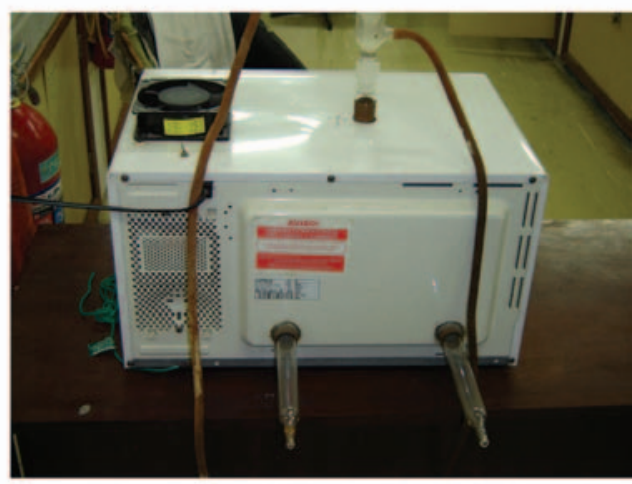

(c)

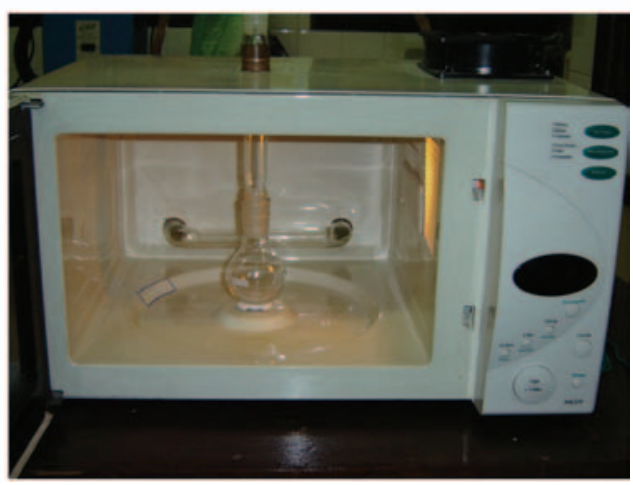

Figura 1. Sistema moderador de ondas. (a) Tubo em " $U$ " e adaptador $24 / 40$ para balão e condensador de refluxo. (b) Foto da face posterior do forno. (c) Tubo em " $U$ " e balão de reação conectados ao forno de microondas

\section{Calibração do aparelho de microondas adaptado}

A determinação da potência do forno de MO foi realizada utilizando-se o método do aumento da temperatura da água. É conhecido que esta elevação de temperatura $(\Delta \mathrm{T})$ é diretamente proporcional à potência da radiação de microondas. Maiores detalhes sobre este procedimento foram recentemente relatados por Nascentes e colaboradores ${ }^{19}$ e Sabadini e colaboradores ${ }^{20}$. Os resultados obtidos neste processo estão mostrados na Tabela 1 e a curva de calibração correspondente está apresentada na Figura 2. Observouse que a potência do forno é crescente em função da temperatura, mas não corresponde ao valor relatado pelo fabricante no manual do aparelho. A posição do ponto ótimo de irradiação no interior do forno foi determinada através de mapeamento do seu interior, utilizando-se papel de fax termo-sensível. Na Figura 1 pode-se observar a localização do ponto ótimo de irradiação.

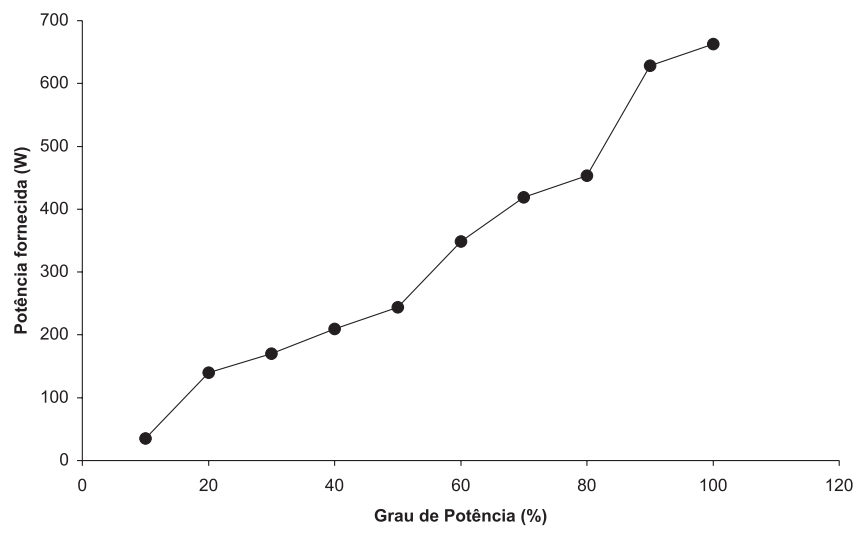

Figura 2. Curva de calibração da potência do aparelho de MO

(a)

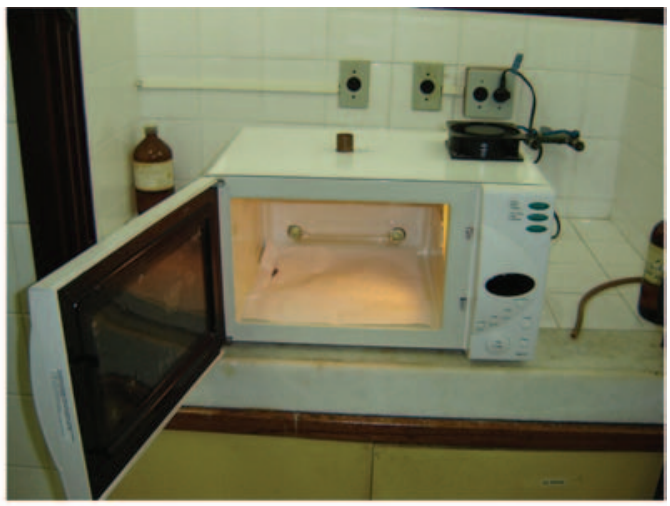

(b)

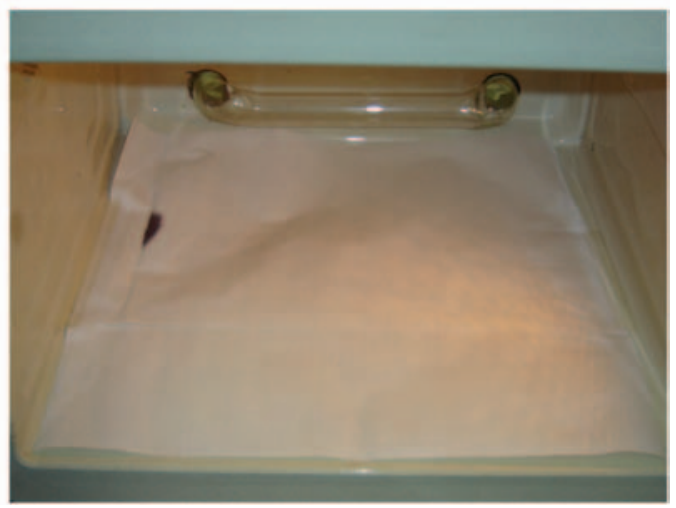

Figura 3. Mapeamento do forno de MO usando papel de fax termo-sensivel. (a) Forramento total da base com folha de papel de fax. (b) Ponto ótimo de irradiação no interior do forno 
Tabela 1. Determinação da potência do forno de MO adaptado

\begin{tabular}{ccc}
\hline $\begin{array}{c}\text { Grau de } \\
\text { Potência }(\%)\end{array}$ & $\begin{array}{c}\text { Variação de } \\
\text { Temperatura }(\mathrm{K})\end{array}$ & $\begin{array}{c}\text { Potência } \\
(\mathrm{W})\end{array}$ \\
\hline 10 & 1,0 & 34,89 \\
20 & 2,0 & 139,56 \\
30 & 4,0 & 169,77 \\
40 & 6,0 & 209,33 \\
50 & 7,0 & 244,22 \\
60 & 10,0 & 348,89 \\
70 & 12,0 & 418,67 \\
80 & 13,0 & 453,56 \\
90 & 18,0 & 628,00 \\
100 & 19,0 & 662,89 \\
\hline
\end{tabular}

\section{Reações de transesterificação catalisadas por argilas}

Nas preparações dos $\beta$-cetoésteres derivados de carboidratos (4a-l) foram utilizadas argilas brasileiras como catalisadores (Esquema 1). As reações sob condições térmicas foram realizadas em manta aquecedora e as efetuadas sob ação das MO mostraram que o forno adaptado é resistente às condições reacionais empregadas.

Inicialmente, preparou-se os acetonídeos dos carboidratos 1a-f ${ }^{21-24}$. Em seguida, foram efetuadas as reações de transesterificação com os $\beta$-cetoésteres etílicos 2 e $\mathbf{3}$. Os acetonídeos foram dissolvidos em tolueno ( $20 \mathrm{~mL} / 1 \mathrm{~g}$ de acetonídeo) e, em seguida, misturados com 1,5 equivalentes de acetoacetato de etila (7) ou benzoilacetato de etila (8) e adicionou-se a argila apropriada. As argilas utilizadas neste trabalho têm sido alvo de diversas pesquisas, tanto sob o ponto de vista de suas propriedades físico-químicas ${ }^{25-26}$, como de sua utilização como catalisadores em reações orgânicas ${ }^{27}$.

No processo térmico de transesterificação, a mistura foi aquecida sob refluxo durante $48 \mathrm{~h}$. Os rendimentos das reações variaram entre 50 e $99 \%$ (Tabela 2). Nas reações com uso de MO, cada mistura de reagentes, em solvente tolueno, foi submetida a sucessivos pulsos de 90 min de MO, na potência máxima do aparelho, durante um período que variou entre 6 a $9 \mathrm{~h}$. Deve-se ressaltar que o marcador de tempo do aparelho só permitia irradiações de no, máximo, 90 min por vez. Em ambos os processos, após as reações de transesterificação, foram efetuadas a filtração da argila e a evaporação do solvente sob pressão reduzida. O óleo resultante foi cromatografado em coluna de sílica

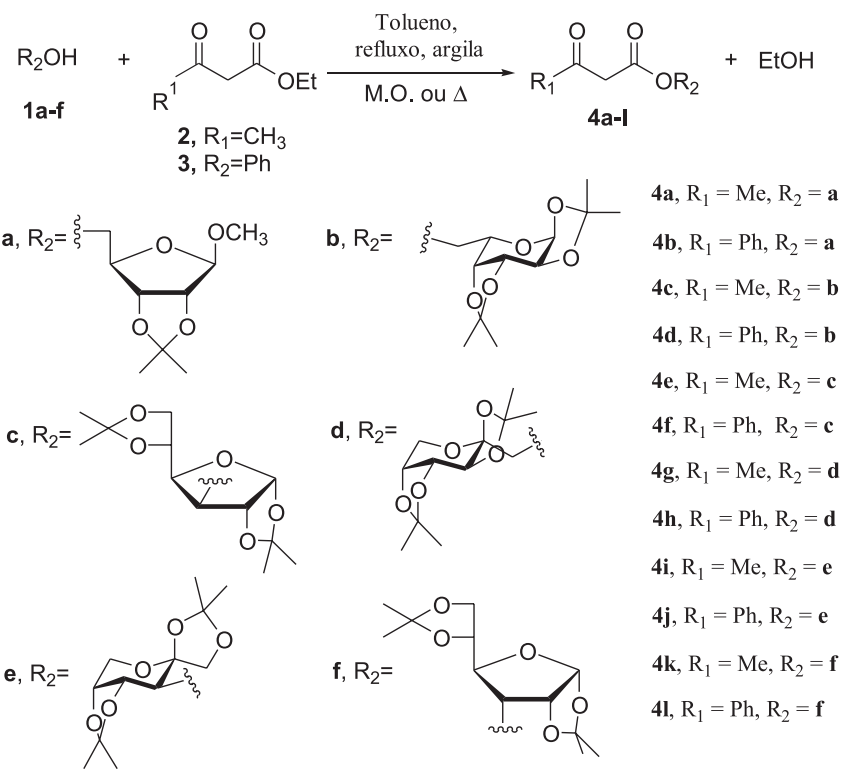

Esquema 1. Esquema reacional utilizado nas transesterificação catalisadas por argilas

gel do tipo "flash", eluindo-se com um gradiente de hexano/ acetato de etila (9:1). Os rendimentos das reações variaram entre 35 e $95 \%$.

Como já verificado em trabalhos anteriores realizados pelo nosso grupo de pesquisa ${ }^{15}$, os rendimentos das reações de transesterificação catalisadas pelas argilas são excelentes (50-99\%), embora o tempo reacional seja muito grande $(48 \mathrm{~h})$. Este tipo de reações quando feito sob irradiação por microondas ocorreu com significantes aumentos de velocidade, sendo o tempo reacional reduzido em até $88 \%$. Observou-se, em ambos os procedimentos, que as reações foram mais eficientes para álcoois primários que secundários, e que seus resultados são dependentes da estrutura do carboidrato. Devese ressaltar que as argilas foram reutilizadas nas diversas reações efetuadas, mantendo sua eficiência, e mostrando que a irradiação de MO não alterou sua estrutura durante o processo.

\section{CONCLUSÃO}

Devido ao alto custo dos fornos de MO comercializados para uso em síntese orgânica, acreditamos que a adaptação de fornos de MO domésticos para este fim, de custo em torno de 300 ve-

Tabela 2. Resultados obtidos nas reações de transesterificação entre 1a-f e $\mathbf{2}$ ou $\mathbf{3}$ catalisadas por argilas em condições térmicas e sob irradiação por microondas

\begin{tabular}{|c|c|c|c|c|c|c|}
\hline \multirow[t]{2}{*}{ Produto } & \multicolumn{3}{|c|}{ Térmico, $\%$ em $48 \mathrm{~h}$} & \multicolumn{3}{|c|}{ Microondas, \% (tempo) } \\
\hline & Esmectita & Atapulgita & Vermiculita & Esmectita & Atapulgita & Vermiculita \\
\hline$\overline{4 a}$ & 73 & 93 & 93 & $70(7 \mathrm{~h})$ & $73(7 \mathrm{~h})$ & $93(7 \mathrm{~h})$ \\
\hline $4 b$ & 72 & 75 & 76 & $65(9 \mathrm{~h})$ & $65(9 \mathrm{~h})$ & $67(9 \mathrm{~h})$ \\
\hline $4 c$ & 76 & 86 & 98 & $76(7 \mathrm{~h})$ & $73(7 \mathrm{~h})$ & $80(7 \mathrm{~h})$ \\
\hline $4 d$ & 89 & 82 & 82 & $70(9 \mathrm{~h})$ & $72(9 \mathrm{~h})$ & $85(9 \mathrm{~h})$ \\
\hline $4 e$ & 50 & 50 & 51 & $52(9 \mathrm{~h})$ & 35 (9 h) & $43(9 \mathrm{~h})$ \\
\hline $4 \mathbf{f}$ & 78 & 80 & 82 & $50(9 \mathrm{~h})$ & 47 (9 h) & $55(9 \mathrm{~h})$ \\
\hline $4 g$ & 98 & 99 & 98 & $73(6 \mathrm{~h})$ & $90(6 \mathrm{~h})$ & $95(6 \mathrm{~h})$ \\
\hline $4 h$ & 91 & 87 & 98 & $60(9 \mathrm{~h})$ & $57(9 \mathrm{~h})$ & $55(9 \mathrm{~h})$ \\
\hline $4 \mathbf{i}$ & 95 & 94 & 84 & $60(7 \mathrm{~h})$ & $70(7 \mathrm{~h})$ & $55(7 \mathrm{~h})$ \\
\hline $4 j$ & 60 & 93 & 99 & $60(9 \mathrm{~h})$ & $60(9 \mathrm{~h})$ & $83(9 \mathrm{~h})$ \\
\hline $4 \mathbf{k}$ & 73 & 96 & 62 & $62(9 \mathrm{~h})$ & $68(9 \mathrm{~h})$ & $63(9 \mathrm{~h})$ \\
\hline 41 & 75 & 90 & 68 & 82 (9 h) & 72 (9 h) & 79 (9 h) \\
\hline
\end{tabular}


zes menor, é perfeitamente viável, face aos experimentos realizados e aos resultados obtidos. O sistema moderador de microondas mostrou-se eficiente e aumentou a vida útil do magnetron. As reações de transesterificação catalisadas por argila e utilizadas como modelo mostraram que a irradiação por MO reduziu significativamente o tempo do processo em todos os casos estudados, quando comparados aos das mesmas reações sob condições térmicas. Dependendo do álcool e da argila empregados nas reações houve redução de até $88 \%$ no tempo reacional, também se utilizando as condições térmicas como parâmetro para comparação.

\section{MATERIAIS E MÉTODOS}

A determinação estrutural das substâncias sintetizadas foi realizada através dos métodos instrumentais de espectroscopia na região de IV e por RMN de ${ }^{1} \mathrm{H}$. Os solventes e reagentes, para fins sintéticos, foram tratados conforme as necessidades requeridas. $\mathrm{O}$ processo de monitoramento das reações foi realizado através da cromatografia em camada fina (c.c.f.), em cromatofolhas de sílica gel 60- F-254, com 0,2 mm de espessura de camada (ref. Merck 5554). Os eluentes foram preparados volume a volume (v/v) e a visualização das substâncias foi efetuada por revelação com reagente sulfato de amônio ou com solução etanólica de vanilina $3 \% \mathrm{em}$ ácido sulfúrico. Para purificação de substâncias por cromatografia em coluna foi utilizada sílica gel $60(0,063-0,200 \mathrm{~mm})$, (ref. Merck 7734). As argilas utilizadas foram obtidas da Fazenda Bravo, Município de Boa Vista, Paraíba, Brasil e foram submetidas a uma série de etapas de preparação envolvendo operações de cominuição, homogeneização e quarteamento. Foram feitas pilhas cônicas e triangulares para homogeneizar o material e, após quarteá-lo em frações, obteve-se a amostra final com massa e granulometria adequadas $(0,1-0,15 \mathrm{~mm})$ à realização das reações.

\section{Adaptação do forno de MO doméstico}

O forno de MO utilizado em todos os experimentos é da marca Eletrolux $^{\circledR}, 110 \mathrm{~V}$, modelo ME27F, $28 \mathrm{~L}$ com gerador de MO na faixa de freqüência de $2450 \mathrm{MHz}$.

Para execução dos experimentos de transesterificação em refluxo sob irradiação de MO foi necessário efetuar-se um furo localizado na parte superior do forno, exatamente no centro de sua cuba. Este furo foi revestido internamente com um anel de cobre (Figura 4). Para adaptação de um condensador de refluxo ao forno foi confeccionado um adaptador de vidro, com $24 \mathrm{~cm}$ de comprimento e com conexões 24/40 (Figura 1). É importante ressaltar que o condensador e o anel de cobre deve estar bem ajustados, para se evitar o vazamento de microondas. Para aumentar a segurança sugere-se que os pontos por onde passam as conexões de vidro, sejam vedados externamente com cola de silicone que, além de impedir o possível vazamento de microondas, é de fácil remoção e resiste a temperaturas superiores a $110^{\circ} \mathrm{C}$. Sugere-se também que os experimentos sejam realizados no interior de uma capela fechada.

Com a finalidade de aumentar a vida útil do magnetron, adaptou-se uma ventoinha de tamanho $12 \times 12 \times 2 \mathrm{~cm}$ acima do magnetron e foram feitos 88 furos de $0,50 \mathrm{~cm}$ de diâmetro na face lateral direita do forno, permitida a saída do ar quente (Figura 5). A alimentação da ventoinha foi feita através da fonte do aparelho $(110 \mathrm{~V})$ e era acionada pelo botão liga/desliga, situado atrás da mesma. Estes dois dispositivos, ventoinha e conjunto de furos, conferiram maior refrigeração ao magnetron, evitando seu desarmamento por superaquecimento pelo (a)

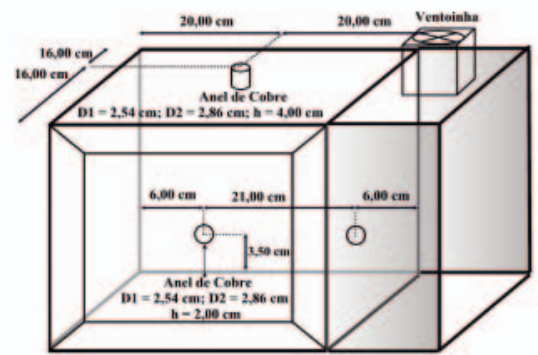

(b)

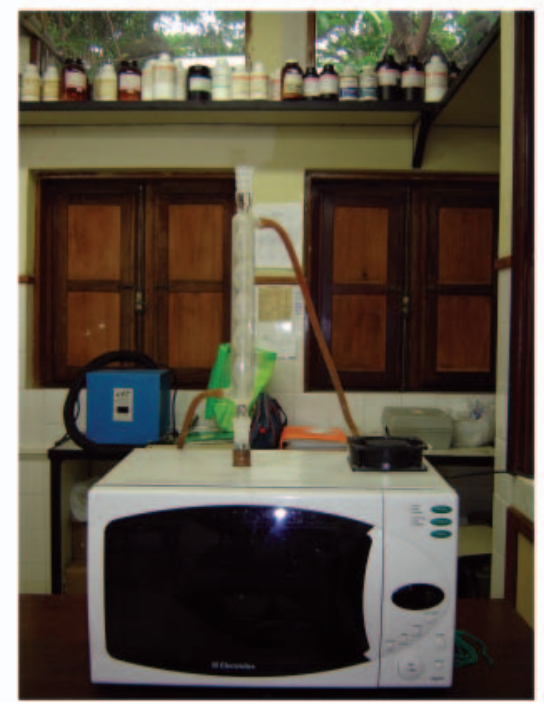

Figura 4. Adaptação de forno de microondas doméstico para reações sob refluxo. (a) diagrama esquemático das furações da cuba e revestimento dos furos com anel de cobre. (b) foto frontal do equipamento de microondas adaptado

termostato do forno (Figura 5). Confeccionou-se um tubo de vidro em forma de "U" (Figura 5) pelo qual se fez passar água, tendo a função de absorver as microondas excedentes. Esta absorção era regulada na medida em que se aumentava ou diminuía o volume de água no tubo, ou se fazia seu avanço ou recuo, tomando como base a face posterior do interior do forno.

\section{Mapeamento da posição do feixe de MO}

A base inferior do forno, sem o prato giratório, foi totalmente coberta com uma folha de papel de fax termo-sensível (Figura 3) e fez-se irradiar microondas na potência $100 \%$ do aparelho, durante $10 \mathrm{~s}$.

\section{Determinação da potência do forno de MO}

A determinação da potência do forno de MO foi realizada pela medida indireta da elevação da temperatura da água por um tempo estabelecido. Um becher de $2 \mathrm{~L}$ contendo $1 \mathrm{~L}$ de água destilada foi posicionado exatamente no ponto assinalado pelo papel de fax (Figura 4). O aquecimento foi realizado por $120 \mathrm{~s}$, nos graus de potência disponíveis no aparelho (10 a 100). A potência (P) foi determinada pela razão entre a energia absorvida pela água $\left(\mathrm{nC}_{\mathrm{p}} \Delta \mathrm{T}\right)$ e o tempo de irradiação $(\mathrm{t})$, conforme a equação $-\mathrm{P}=$ $\mathrm{nC} \Delta \mathrm{T} / \mathrm{t}$ - na qual $\mathrm{n}$ é o número de mols de água, neste caso 55,6 mols; $\mathrm{C}_{\mathrm{p}}$, a capacidade calorífica molar da água $\left(75,3 \mathrm{~J} \mathrm{~K}^{-1} \mathrm{~mol}\right.$ $\left.{ }^{1}\right) ; \Delta \mathrm{T}$, a variação de temperatura observada e t, o tempo de irradiação $(120 \mathrm{~s})$. 
(a)

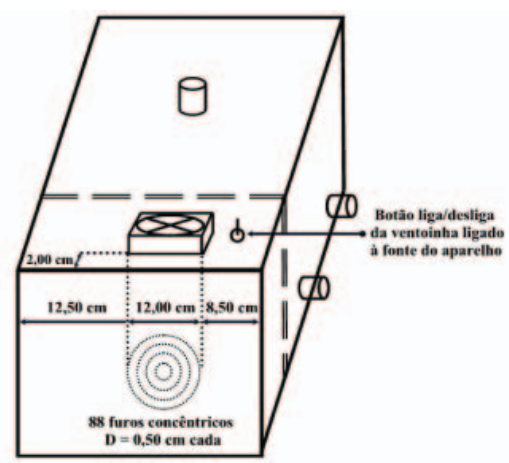

(b)

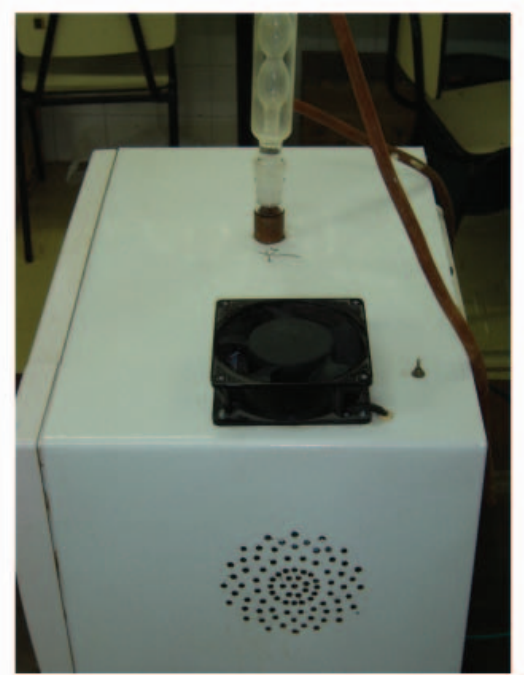

Figura 5. Adaptação do sistema de refrigeração do magnetron. (a) Adaptação da ventoinha sobre o magnetron e dos furos laterais para circulação de ar. (b) foto lateral do sistema de refrigeração do microondas

\section{Método geral de transesterificação}

As reações de transesterificação entre os $\beta$-cetoésteres etílicos (2 e 3) e acetonídeos (1a-f) foram realizadas em tolueno $(20 \mathrm{~mL} / 1$ g de acetonídeo) ao qual se adicionou 1,5 equivalentes de acetoacetato de etila (2) ou benzoilacetato de etila (3), 1 equivalente do acetonídeo e $20 \%$ em peso da argila apropriada (em relação ao acetonídeo). Após os tempos indicados na Tabela 2, a argila foi removida por filtração, o solvente foi evaporado sob pressão reduzida e o produto bruto resultante foi cromatografado em coluna de gel de sílica utilizando-se mistura de eluentes de polaridade crescente entre hexano/acetato de etila. Os rendimentos das reações estão mostrados na Tabela 2. Os $\beta$-cetoésteres 4a-l foram analisados por métodos espectroscópicos de $\mathrm{RMN}$ de ${ }^{1} \mathrm{H}$ e por IV e os dados estão de acordo aqueles descritos na literatura ${ }^{15}$.

\section{AGRADECIMENTOS}

V. F. Ferreira e M. C. B. V. de Souza agradecem ao CNPq pelas bolsas de produtividade e pelos apoios financeiros do $\mathrm{CNPq}$, FAPERJ, UFF e PROAP-CAPES. F. C. da Silva agradece à CAPES pela bolsa de doutorado concedida ao Programa de Pós-Graduação em Química Orgânica da UFF. Os autores agradecem ao Prof. C. Zucco (UFSC) pelas suas valiosas sugestões e informações técnicas e ao Sr. R. L. da Silva pelas adaptações realizadas no forno.

\section{REFERÊNCIAS}

1. Lew, A.; Krutzik, P. O.; Hart, M. E.; Chamberlin, A. R.; J. Comb. Chem. 2002, 4, 95 .

2. Lidström, P.; Tierney, J.; Wathey, B.; Westman, J.; Tetrahedron 2001, 57, 9225.

3. Larhed, M.; Moberg, C.; Hallberg, A.; Acc. Chem. Res. 2002, 35, 717.

4. Sanseverino, A. M.; Quim. Nova 2002, 25, 660.

5. Varma, R. S.; Pure Appl. Chem. 2001, 73, 193.

6. Graebin, C. S.; Eifler-Lima, V. L.; Quim. Nova 2005, 28, 73 e referências citadas.

7. Carmosini, N.; Ghoreshy, S.; Koether, M. C.; J. Chem. Educ. 1997, 74, 986.

8. Arruda, M. A. Z.; Santelli, R. E.; Quim. Nova 1997, 20, 638.

9. Pecoraro, E.; Davolos, M. R.; Jafelicci Jr., M. R.; Quim. Nova 1997, 20, 89.

10. Ferreira, V. F.; Souza, M. C. V. S.; Rianelli, R. S.; Silva, F. C.; Antunes, O. A. C. Em Progress in Catalisys Research; Bevy, L. P., ed.; Nova Science Publishers: New York, 2005, cap. 7

11. Vaccari, A.; Appl. Clay Sci. 1999, 14, 161.

12. Yadav, J. S.; Reddy, S.; Kumar, G. M.; Merthy, Ch. V. S. R.; Tetrahedron Lett. 2001, 42, 89; Vodnár, J.; Farkas, J.; Békássy, S.; Appl. Catal., A 2001, 208, 329; Rigo, B.; Waterlot, C.; Couturier, D.; Tetrahedron Lett. 2000, 41, 317; Choudary, B. M.; Choudary, N. S.; Kantam, L. M.; Tetrahedron 2000, 56, 7291; Sartori, G.; Maggi, R.; Conforti, M. L.; Bigi, F.; Tetrahedron 2000, 56, 2709; Campanati, M.; Vaccari, A.; Catal. Today 2000, 60, 289.

13. Díaz, F. R. V.; Santos, P. S.; Quim. Nova 2001, 24, 345.

14. Otera, J.; Chem. Rev. 1993, 93, 1449.

15. Ferreira, V. F.; Pereira, W. C.; Silva, F. C.; Rianelli, R. S.; Tetrahedron Lett. 2002, 43, 1165 e referências citadas.

16. Braibante, H. T. S.; Braibante, M. E. F.; Rosso, G. B.; Oriques, D. A .; J. Braz. Chem. Soc. 2003, 14, 994.

17. Silva, F. C.; de Souza, M. C. B. V.; Ferreira, V. F.; Resumos da $27^{a}$ Reunião Anual da Sociedade Brasileira de Química, Salvador, Brasil, 2004.

18. Ardon, M.; Hayes, P. D.; Hogartha, G.; J. Chem. Educ. 2002, 79, 1249.

19. Rosini, F.; Nascentes, C. C.; Nóbrega, J. A.; Quim. Nova 2004, 27, 1012.

20. Barboza, A. C. R. N.; Cruz, C. V. M. S.; Graziani, M. B.; Lorenzetti, M. C. F.; Sabadini, E.; Quim. Nova 2001, 24, 901.

21. Verhart, C, G. J.; Carls, B. M. G.; Zwanenburg, B.; Chittenden, G. J. F.; Rec. Trav. Chim. Pays-Bas 1992, 111, 348.

22. Silva, F. C.; Ferreira, V. F.; Perrone, C. C.; Quim. Nova 2001, 24, 905.

23. Fisher, E.; Chem. Ber. 1895, 28, 1145.

24. Sowa, W.; Thomas, G. H. S.; Can. J. Chem. 1966, 44, 836.

25. Araujo, A.; de Santos, O.; Cerâmica 1986, 32, 339.

26. Díaz, F. R.; Cerâmica 1986, 32, 319.

27. Rianelli, R. S.; de Souza, M. C. B. V.; Ferreira, V. F.; Synth. Commun. 2004, 34,951 . 